\title{
LOWER BOUNDS FOR ENTIRE FUNCTIONS
}

\author{
O.A. KRIVOSHEEVA, A.S. KRIVOSHEEV, A.I. RAFIKOV
}

\begin{abstract}
We study lower bounds for entire functions of proximate order and of completely regular growth. We introduce the notion of the index condensation for sequences of complex numbers of proximate order. This notion generalizes that of the index of condensation for sequences of order one. We also introduce a properly balanced set, which is a properly distributed set with a zero condensation index. We show that a regular set is properly balanced and we prove that the properly balanced property of the zero set of an entire function is a necessary and sufficient condition for the existence of family of pairwise disjoint circles with the centers at its zeros and with relatively small radii. Outside these circles, the absolute value of the function admits lower bounds asymptotically coinciding with its upper bounds in the entire plane. Thus, we show that the notion of a properly balanced set naturally generalizes the notion of a regular set in the case of arbitrary sequences including multiples sequences. A method for constructing of an exceptional set consisting of circles with centers at zeroes of entire function is also provided. In some cases, we can make the sum of the radii of these circles arbitrarily small.
\end{abstract}

Keywords: Entire function, proximate order, completely regular growth, properly balanced set, regular set.

Mathematics Subject Classification: 30D10

\section{INTRODUCTION}

Let $f$ be an entire function of proximate order $\rho(r)$ and of regular growth [1, Ch. III], $\Lambda=\left\{\lambda_{k}, n_{k}\right\}_{k=1}^{\infty}$ be its multiple zero set, where $n_{k}$ stands for the multiplicity of the zero $\lambda_{k}$. In the work, we study lower bounds for the function $f$, which holds in the entire plane except for a special set of circles centered at the points $\lambda_{k}$.

A classical result by B.Ya. Levin [1, Ch. II, Thm. 2, Ch. III, Thm. 4] established a closed connection between the behavior of entire functions of regular growth $f$ and their zero sets $\Lambda$. At that, there were obtained lower bounds for the function $|f|$ outside an exceptional $C_{0}$-set [1, Ch. II, Sect. 1]. By an opinion of B.Ya. Levin [1, Ch. II, Sects. 1, 6], a disadvantage of the exceptional set of the circles is that it is not constructed effectively, namely, it is not said how to construct $C_{0}$-set. Because of this, B.Ya. Levin introduced the notion of a regular set consisting of simple points $\lambda_{k}$ (as $n_{k}=1$ ). In this case, it is possible to provide a full description of exceptional circles being disjoint circles centered at $\lambda_{k}$ of special radii. However, the set obtained in such way is not a $C_{0}$-set although its linear density can be made arbitrarily small.

The regularity of the set means that its points are separated in a special way. This is why a function $f$ with regular zero set $\Lambda=\left\{\lambda_{k}, 1\right\}_{k=1}^{\infty}$ possesses nice lower bounds outside an exceptional set formed by disjoint circles $B\left(\lambda_{k}, \gamma_{k}\right)$, whose radii are relatively small, namely, are infinitesimal in comparison with the centers as $k \rightarrow \infty$ [1, Ch. II, Sect. 1, Thm. 5].

O.A. Krivosheeva, A.S. Krivosheev, A.I. Rafikov, Lower bounds for entire functions.

(C)Krivosheeva O.A., Krivosheev A.S., Rafikov A.I. 2019.

Submitted June 10, 2019.

The reported study of the first author was funded by RFBR according to the research project no. 18-31-00029. 
In the present work we employ another tool allowing us to separate points $\lambda_{k}$, a condensation index $S_{\Lambda}$ of a sequence $\Lambda$ introduced in [2]. This index is appropriate for working with arbitrary sequences including multiple ones. It is considered in the second section.

The third section is devoted to obtaining lower bounds for special polynomials defined by the sequence $\Lambda=\left\{\lambda_{k}, 1\right\}_{k=1}^{\infty}$ of normalized in a special way pieces of an infinite product representing an entire function with a multiple zero set $\Lambda$. We study the relations between these estimates and condensation indices. We construct exceptional circles centered at the points $\lambda_{k}$, outside which the mentioned polynomials admit appropriate lower bounds.

On the base of the results of the third section, in the fourth section we study lower bounds for entire functions of a proximate order $\rho(r)$ and of completely regular growth. We introduce the notion of a properly balanced set, which is a properly distributed set with the zero condensation index $S_{\Lambda}$. We show that a regular set is properly balanced, see Theorem 4.2 . We also prove that a properly balanced property of the zero set $\Lambda=\left\{\lambda_{k}, 1\right\}_{k=1}^{\infty}$ of a function $f$ is a necessary and sufficient condition ensuring the existence of a family of mutually disjoint circles with centers at the points $\lambda_{k}$ and relatively small radii, outside which the function $|f|$ admit lower bounds coinciding asymptotically with its upper bounds in the entire plane, see Theorem 4.5. Thus, we show that the notion of a properly balanced set is a natural generalization of the notion of a regular set for the case of arbitrary sequences including the multiple ones. We provide an effective algorithm for constructing an exceptional set formed by circles centered at the points $\lambda_{k}$, see Theorem 4.3.

\section{Condensation indeX}

Let $\Lambda=\left\{\lambda_{k}, 1\right\}_{k=1}^{\infty}$ be a sequence of different complex numbers $\lambda_{k}$ and their multiplicities $n_{k}$. We assume that $\left|\lambda_{k}\right|$ does not decrease and $\left|\lambda_{k}\right| \rightarrow \infty, k \rightarrow \infty$. We consider sequences of a proximate order $\rho(r)$ [1, Ch. I, Sect. 12]. We recall main properties of $\rho(r)$. A function $\rho(r)$, $r>0$, satisfying the conditions

$$
\lim _{r \rightarrow \infty} \rho(r)=\rho>0, \quad \lim _{r \rightarrow \infty} r \rho^{\prime}(r) \ln r=0,
$$

is called a proximate order. We have

$$
\left(r^{\rho(r)}\right)^{\prime}=r^{\rho(r)}\left(\rho^{\prime}(r) \ln r+\frac{\rho(r)}{r}\right)=r^{\rho(r)-1}\left(r \rho^{\prime}(r) \ln r+\rho(r)\right) .
$$

Thus, by (2.1), the function $r^{\rho(r)}$ increases for sufficiently large $r$. We let $L(r)=r^{\rho(r)-\rho}$. The function $L(r)$ is slowly growing [1, Ch. I, Sect. 12, Lm. 5], that is,

$$
\lim _{r \rightarrow \infty} \frac{L(c r)}{L(r)}=1
$$

uniformly on each segment $0<a \leqslant c \leqslant b<\infty$. It follows from (2.2) that for each $\varepsilon>0$ and each $c \in[a, b]$, the inequality holds:

$$
(1-\varepsilon) c^{\rho} r^{\rho(r)} \leqslant(c r)^{\rho(c r)} \leqslant(1+\varepsilon) c^{\rho} r^{\rho(r)}, \quad r \geqslant R(\varepsilon) .
$$

A sequence $\Lambda$ is said to have a finite upper density $\bar{n}(\Lambda)$ (at order $\rho(r)$ ) if

$$
\bar{n}(\Lambda)=\varlimsup_{r \rightarrow \infty} \frac{n(r, \Lambda)}{r^{\rho(r)}}<\infty,
$$

where $n(r, \Lambda)$ is the amount of the points $\lambda_{k}$ counting their multiplicities $n_{k}$ in the circle $B(0, r)$ of radius $r$ centered at the origin. We introduce a local characteristics of the sequence $\Lambda$. Following work [2], we let

$$
q_{\Lambda}(z, w, \delta)=\prod_{\lambda_{k} \in B(w, \delta|w|)}\left(\frac{z-\lambda_{k}}{3 \delta\left|\lambda_{k}\right|}\right)^{n_{k}}
$$


As the ball $B(w, \delta|w|)$ contains none of $\lambda_{k}$, we let $q_{\Lambda}(\lambda, w, \delta) \equiv 1$. The absolute value of $q_{\Lambda}(\lambda, w, \delta)$ can be interpreted as a measure of accumulation of the points $\lambda_{k} \in B(w, \delta|w|)$ at $\lambda$. By its meaning, the quantity $\frac{\ln \left|q_{\Lambda}(\lambda, w, \delta)\right|}{|w|}$ is similar to the logarithm of the geometric mean (arithmetic mean of the logarithms) of the normed distances from the points $\lambda_{k} \in B(w, \delta|w|$ ) to the point $\lambda$. If $\delta \in(0,1)$, the absolute value of each factor $q_{\Lambda}$ in the circle $B(w, \delta|w|)$ is bounded from above by the quantity $2(3(1-\delta))^{-1}$. This is why

$$
\frac{\left|\lambda-\lambda_{k}\right|}{3 \delta\left|\lambda_{k}\right|} \leqslant 1, \quad \lambda, \lambda_{k} \in B(w, \delta|w|), \quad \delta \in\left(0, \frac{1}{3}\right)
$$

Moreover, the inequality holds:

$$
\frac{\left|\lambda-\lambda_{k}\right|}{3 \delta\left|\lambda_{k}\right|} \geqslant 1, \quad \lambda \in B(w, 5 \delta|w|), \quad \lambda_{k} \in B(w, \delta|w|), \quad \delta \in\left(0, \frac{1}{3}\right)
$$

We introduce extra functions, see [2]. We let

$$
q_{\Lambda}^{m}(z, \delta)=\prod_{\lambda_{k} \in B\left(\lambda_{m}, \delta\left|\lambda_{m}\right|\right), k \neq m}\left(\frac{z-\lambda_{k}}{3 \delta\left|\lambda_{k}\right|}\right)^{n_{k}}=q_{\Lambda}(z, w, \delta)\left(\frac{z-\lambda_{m}}{3 \delta\left|\lambda_{m}\right|}\right)^{n_{m}}, \quad m \geqslant 1 .
$$

Following [2], we define the condensation index:

$$
S_{\Lambda}=\lim _{\delta \rightarrow 0} S_{\Lambda}(\delta), \quad S_{\Lambda}(\delta)=\varliminf_{m \rightarrow \infty} \frac{\ln \left|q_{\Lambda}^{m}\left(\lambda_{m}, \delta\right)\right|}{\left|\lambda_{m}\right|^{\rho\left(\left|\lambda_{m}\right|\right)}}
$$

It follows from (2.4) that the limit is well-defined and $S_{\Lambda} \leqslant 0$. Various examples on calculating the index $S_{\Lambda}$ in the case $\rho(r) \equiv 1$ were given in works [3]- $[5]$. We consider extra examples related with the notion of a regular set. Let $\Lambda=\left\{\lambda_{k}, 1\right\}$. Assume that one of the following conditions holds [1, Ch. II, Sect. 1]:

(C) There exists a number $d>0$ such that the circles of the radii

$$
r_{k}=d\left|\lambda_{k}\right|^{1-\frac{\rho\left(\left|\lambda_{k}\right|\right)}{2}}
$$

centered at the points $\lambda_{k}$ are mutually disjoint.

(C') The points $\lambda_{k}$ are located in finitely many closed angles with a common vertex at the origin having no other common points. At that, for all points $\lambda_{k(p)}$ located in a same angle, the inequality

$$
\left|\lambda_{k(p+1)}\right|-\left|\lambda_{k(p)}\right| \geqslant d\left|\lambda_{k(p)}\right|^{1-\rho\left(\left|\lambda_{k(p)}\right|\right)}, \quad d>0
$$

holds true.

Let us show that in both cases $S_{\Lambda}=0$. First we assume that Condition (C') is satisfied. We begin with observing that for sufficiently small $\delta>0\left(\delta<\delta_{0}<\frac{1}{3}\right)$ and $k \geqslant k_{0}$ the circle $B\left(\lambda_{k}, \delta\left|\lambda_{k}\right|\right)$ intersects just one of the angles mentioned in the formulation of Condition (C'); we denote them by $\Gamma_{j}$. This is why it follows from the definition of the quantity $S_{\Lambda}$ that it is sufficient to prove the identity $S_{\Lambda_{j}}=0$, where $\Lambda_{j}$ is a part of the sequence $\Lambda$ located in the angle $\Gamma_{j}$. We denote $\Lambda_{j}=\left\{\lambda_{k(p)}, 1\right\}_{p=1}^{\infty}$.

Let $\delta \in\left(0, \delta_{0}\right)$ and $k(p) \geqslant k_{0}$. By (2.3) and Condition (C'), there exists $\alpha>0$ such that

$$
\left|\lambda_{k(s+1)}\right|-\left|\lambda_{k(s)}\right| \geqslant d\left|\lambda_{k(s)}\right|^{1-\rho\left(\left|\lambda_{k(s)}\right|\right)} \geqslant \alpha\left|\lambda_{k(p)}\right|^{1-\rho\left(\left|\lambda_{k(p)}\right|\right)}=h_{p}, \quad \lambda_{k(s)} \in B\left(\lambda_{k(p)}, \delta\left|\lambda_{k(p)}\right|\right) .
$$


Then by (2.4) we get

$$
\begin{aligned}
\sum_{\lambda_{k(s)} \in B\left(\lambda_{k(p)}, \delta\left|\lambda_{k(p)}\right|\right), s \neq p} \ln \frac{\left|\lambda_{k(s)}-\lambda_{k(p)}\right|}{3 \delta\left|\lambda_{k(s)}\right|} & =\sum_{\lambda_{k(s)} \in B\left(\lambda_{k(p)}, \delta\left|\lambda_{k(p)}\right|\right),\left|\lambda_{k(s)}\right|<\left|\lambda_{k(p)}\right|} \ln \frac{\left|\lambda_{k(s)}-\lambda_{k(p)}\right|}{3 \delta\left|\lambda_{k(s)}\right|} \\
& +\sum_{\lambda_{k(s)} \in B\left(\lambda_{k(p)}, \delta\left|\lambda_{k(p)}\right|\right),\left|\lambda_{k(s)}\right|>\left|\lambda_{k(p)}\right|} \ln \frac{\left|\lambda_{k(s)}-\lambda_{k(p)}\right|}{3 \delta\left|\lambda_{k(s)}\right|} \\
\geqslant & 2 \sum_{l=1}^{l_{p}} \ln \frac{l h_{p}}{3 \delta(1+\delta)\left|\lambda_{k(p)}\right|},
\end{aligned}
$$

where $l_{p}$ is an integer part of the number $\frac{\delta\left|\lambda_{k(p)}\right|}{h_{p}}$. This yields

$$
\begin{aligned}
\frac{1}{\left|\lambda_{k(p)}\right|^{\rho\left(\left|\lambda_{k(p)}\right|\right)}} \sum_{\lambda_{k(s)} \in B\left(\lambda_{k(p)}, \delta\left|\lambda_{k(p)}\right|\right), s \neq p} \ln \frac{\left|\lambda_{k(s)}-\lambda_{k(p)}\right|}{3 \delta\left|\lambda_{k(s)}\right|} & \geqslant \frac{2}{\left|\lambda_{k(p)}\right|^{\rho\left(\left|\lambda_{k(p)}\right|\right)}} \ln \left(\frac{\left(l_{p}\right) !\left(h_{p}\right)^{l_{p}}}{\left.3 \delta(1+\delta)\left|\lambda_{k(p)}\right|\right)^{l_{p}}}\right) \\
& \geqslant \frac{2}{\left|\lambda_{k(p)}\right|^{\rho\left(\left|\lambda_{k(p)}\right|\right)}} \ln \left(\frac{\left(h_{p} l_{p}\right)^{l_{p}}}{\left(12 \delta\left|\lambda_{k(p)}\right|\right)^{l_{p}}}\right) \\
& =\frac{2 l_{p}}{\left|\lambda_{k(p)}\right|^{\rho\left(\left|\lambda_{k(p)}\right|\right)}} \ln \frac{h_{p} l_{p}}{12 \delta\left|\lambda_{k(p)}\right|} .
\end{aligned}
$$

Hence,

$$
\varliminf_{p \rightarrow \infty} \frac{1}{\left|\lambda_{k(p)}\right|^{\rho\left(\left|\lambda_{k(p)}\right|\right)}} q_{\Lambda}^{k(p)}\left(\lambda_{k(p)}, \delta\right) \geqslant-2 \frac{\delta}{\alpha} \ln 12 .
$$

This implies the identity $S_{\Lambda_{j}}=0$.

Assume now that Condition (C) holds. We fix $\delta \in\left(0, \frac{1}{3}\right)$. By (2.3), there exists numbers $\alpha, \beta>0$ such that

$$
\alpha d\left|\lambda_{k}\right|^{1-\frac{\rho\left(\left|\lambda_{k}\right|\right)}{2}} \leqslant d\left|\lambda_{s}\right|^{1-\frac{\rho\left(\left|\lambda_{k}\right|\right)}{2}} \leqslant \beta d\left|\lambda_{k}\right|^{1-\frac{\rho\left(\left|\lambda_{k}\right|\right)}{2}}, \quad \lambda_{s} \in B\left(\lambda_{k}, \delta\left|\lambda_{k}\right|\right) .
$$

This is why, it follows from Condition (C) that the circle $B\left(\lambda_{k}, d\left|\lambda_{k}\right|^{1-\frac{\rho\left(\left|\lambda_{k}\right|\right)}{2}}\right)$ contains no points $\lambda_{s}, s \neq k$, and the amount $m_{n, k}$ of the points $\lambda_{s} \in B\left(\lambda_{k}, \delta\left|\lambda_{k}\right|\right)$ located in the annulus

$$
K_{n, k}=B\left(\lambda_{k},(n+1) d\left|\lambda_{k}\right|^{1-\frac{\rho\left(\left|\lambda_{k}\right|\right)}{2}}\right) \backslash B\left(\lambda_{k}, n d\left|\lambda_{k}\right|^{1-\frac{\rho\left(\left|\lambda_{k}\right|\right)}{2}}\right), \quad n \geqslant 1,
$$

does not exceeds the quotient of the annulus' area

$$
B\left(\lambda_{k},((n+1)+\beta) d\left|\lambda_{k}\right|^{1-\frac{\rho\left(\left|\lambda_{k}\right|\right)}{2}}\right) \backslash B\left(\lambda_{k},(n-\beta) d\left|\lambda_{k}\right|^{1-\frac{\rho\left(\left|\lambda_{k}\right|\right)}{2}}\right)
$$

and the area of the circle $B\left(\lambda_{s}, d\left|\lambda_{s}\right|^{1-\frac{\rho\left(\left|\lambda_{k}\right|\right)}{2}}\right)$ of a minimal radius for the same $\lambda_{s}$. The area of this circle is estimated from below by $\pi\left(\alpha d\left|\lambda_{k}\right|^{1-\frac{\rho\left(\left|\lambda_{k}\right|\right)}{2}}\right)^{2}$. We have

$$
m_{n, k} \leqslant \frac{\pi\left(((n+1)+\beta)^{2}-(n-\beta)^{2}\right) d^{2}\left|\lambda_{k}\right|^{2-\rho\left(\left|\lambda_{k}\right|\right)}}{\pi \alpha^{2} d^{2}\left|\lambda_{k}\right|^{2-\rho\left(\left|\lambda_{k}\right|\right)}}=\frac{(2 n+1)(2 \beta+1)}{\alpha^{2}} .
$$

Let $j_{k}$ be an integer part of the number $\frac{\delta\left|\lambda_{k}\right|}{d\left|\lambda_{k}\right|^{1-\frac{\rho\left(\lambda_{k} \mid\right)}{2}}}$. Then

$$
m_{n, k} \leqslant \frac{\left(2 j_{k}+1\right)(2 \beta+1)}{\alpha^{2}}=p_{k}, \quad 1 \leqslant n \leqslant j_{k} .
$$

Taking into consideration the inequality

$$
\left|\lambda_{s}-\lambda_{k}\right| \geqslant n d\left|\lambda_{k}\right|^{1-\frac{\rho\left(\left|\lambda_{k}\right|\right)}{2}}, \quad \lambda_{s} \in K_{n, k}, \quad 1 \leqslant n \leqslant j_{k}
$$


we obtain

$$
\sum_{\lambda_{s} \in B\left(\lambda_{k}, \delta\left|\lambda_{k}\right|\right), s \neq k} \ln \frac{\left|\lambda_{s}-\lambda_{k}\right|}{3 \delta\left|\lambda_{s}\right|} \geqslant \sum_{n=1}^{j_{k}} \ln \frac{n p_{k} d\left|\lambda_{k}\right|^{1-\frac{\rho\left(\left|\lambda_{k}\right|\right)}{2}}}{4 \delta\left|\lambda_{k}\right|} \geqslant j_{k} \ln \left(\frac{p_{k} j_{k} d\left|\lambda_{k}\right|^{1-\frac{\rho\left(\left|\lambda_{k}\right|\right)}{2}}}{12 \delta\left|\lambda_{k}\right|}\right) .
$$

Thus,

$$
\begin{aligned}
S_{\Lambda}(\delta) & =\varliminf_{k \rightarrow \infty} \frac{1}{\left|\lambda_{k}\right|^{\rho\left(\left|\lambda_{k}\right|\right)}} q_{\Lambda}^{k}\left(\lambda_{k}, \delta\right) \geqslant \varliminf_{k \rightarrow \infty} \frac{j_{k}}{\left|\lambda_{k}\right|^{\rho\left(\left|\lambda_{k}\right|\right)}} \ln \left(\frac{p_{k} j_{k} d\left|\lambda_{k}\right|^{1-\frac{\rho\left(\left|\lambda_{k}\right|\right)}{2}}}{12 \delta\left|\lambda_{k}\right|}\right) \\
& =\varliminf_{k \rightarrow \infty} \frac{\delta}{d\left|\lambda_{k}\right|^{\frac{\rho\left(\left|\lambda_{k}\right|\right)}{2}}} \ln j_{k}=\lim _{k \rightarrow \infty} \frac{\delta}{d\left|\lambda_{k}\right|^{\frac{\rho\left(\left|\lambda_{k}\right|\right)}{2}}} \ln \frac{\delta}{d\left|\lambda_{k}\right|^{-\frac{\rho\left(\left|\lambda_{k}\right|\right)}{2}}}=0 .
\end{aligned}
$$

Therefore, $S_{\Lambda}=0$.

\section{LOWER BOUNDS FOR SPECIAL POLYNOMIALS}

We provide some results concerning the index $S_{\Lambda}$; we shall $\mathrm{n}$ need them in what follows.

Lemma 3.1. Let $\Lambda=\left\{\lambda_{k}, n_{k}\right\}, S_{\Lambda}=0$, and $B$ be an open set containing all points $\lambda_{k}$. The following statements are equivalent:

1. For each $\varepsilon>0$, there exist number $R>0$ and $\delta \in\left(0, \frac{1}{3}\right)$ such that

$$
\ln \left|q_{\Lambda}(z, w, \delta)\right| \geqslant-\varepsilon|z|^{\rho(|z|)}, \quad z \in \mathbb{C} \backslash B, \quad|w| \geqslant R .
$$

2. For each $\varepsilon>0$, there exist numbers $R>0$ and $\delta \in\left(0, \frac{1}{3}\right)$ such that

$$
\ln \left|q_{\Lambda}(z, w, \delta)\right| \geqslant-\varepsilon|z|^{\rho(|z|)}, \quad z \in B(w, \delta|w|) \cap \partial B, \quad|w| \geqslant R .
$$

3. For each $\varepsilon>0$ there exists a number $k_{0}$ and a number $\delta \in\left(0, \frac{1}{3}\right)$ such that for each $k \geqslant k_{0}$ the inequality holds:

$$
\ln \left|\frac{z-\lambda_{k}}{3 \delta\left|\lambda_{k}\right|}\right|^{n_{k}} \geqslant-\varepsilon\left|\lambda_{k}\right|^{\rho\left(\left|\lambda_{k}\right|\right)}, \quad z \in B\left(\lambda_{k}, \delta\left|\lambda_{k}\right|\right) \cap \partial B .
$$

4. For each $\varepsilon>0$ there exists a number $k_{0}$ and a number $\delta \in\left(0, \frac{1}{3}\right)$ such that for each $k \geqslant k_{0}$ the inequality holds:

$$
\ln \left|\frac{z-\lambda_{k}}{3 \delta\left|\lambda_{k}\right|}\right|^{n_{k}} \geqslant-\varepsilon|z|^{\rho(|z|)}, \quad z \in \mathbb{C} \backslash B .
$$

Proof. Assume that (3.1) holds. Then (3.2) is obvious. Since $|z| \leqslant(1+\delta)\left|\lambda_{k}\right|$ as $z \in B\left(\lambda_{k}, \delta\left|\lambda_{k}\right|\right)$, in view of the definition of the function $q_{\Lambda}(z, w, \delta)$ and $(2.4),(3.2),(2.3)$, we obtain:

$$
\ln \left|\frac{z-\lambda_{k}}{3 \delta\left|\lambda_{k}\right|}\right|^{n_{k}} \geqslant \ln \left|q_{\Lambda}\left(z, \lambda_{k}, \delta\right)\right| \geqslant-\varepsilon|z|^{\rho(|z|)} \geqslant-\varepsilon\left(2\left|\lambda_{k}\right|\right)^{\rho\left(2\left|\lambda_{k}\right|\right)} \geqslant-2^{\rho+1} \varepsilon\left|\lambda_{k}\right|^{\rho\left(\left|\lambda_{k}\right|\right)},
$$

where $k \geqslant k_{1}$ and $z \in B\left(\lambda_{k}, \delta\left|\lambda_{k}\right|\right) \cap \partial B$. This gives (3.3).

Assume (3.3) and let us prove (3.4). We take $\varepsilon>0$ and we find $k_{0}$ and $\delta_{0} \in\left(0, \frac{1}{3}\right)$ such that

$$
\ln \left|\frac{z-\lambda_{k}}{3 \delta_{0}\left|\lambda_{k}\right|}\right|^{n_{k}} \geqslant-\varepsilon\left|\lambda_{k}\right|^{\rho\left(\left|\lambda_{k}\right|\right)}, \quad z \in B\left(\lambda_{k}, \delta_{0}\left|\lambda_{k}\right|\right) \cap \partial B, \quad k \geqslant k_{0} .
$$

We let $\delta=\frac{\delta_{0}}{3}$ and we have

$$
\ln \left|\frac{z-\lambda_{k}}{3 \delta\left|\lambda_{k}\right|}\right|^{n_{k}} \geqslant \ln \left|\frac{z-\lambda_{k}}{3 \delta_{0}\left|\lambda_{k}\right|}\right|^{n_{k}} \geqslant-\varepsilon\left|\lambda_{k}\right|^{\rho\left(\left|\lambda_{k}\right|\right)}, \quad z \in B\left(\lambda_{k}, 3 \delta\left|\lambda_{k}\right|\right) \cap \partial B, \quad k \geqslant k_{0} .
$$

If $z \in \mathbb{C} \backslash B\left(\lambda_{k}, 3 \delta\left|\lambda_{k}\right|\right)$, then

$$
\ln \left|\frac{z-\lambda_{k}}{3 \delta\left|\lambda_{k}\right|}\right|^{n_{k}} \geqslant 0>-\varepsilon|z|^{\rho(|z|)} .
$$


In view of the above arguing and the minimum principle for the harmonic functions we obtain:

$$
\ln \left|\frac{z-\lambda_{k}}{3 \delta\left|\lambda_{k}\right|}\right|^{n_{k}} \geqslant-\varepsilon\left|\lambda_{k}\right|^{\rho\left(\left|\lambda_{k}\right|\right)}, \quad z \in B\left(\lambda_{k}, 3 \delta\left|\lambda_{k}\right|\right) \backslash B, \quad k \geqslant k_{0} .
$$

Let $z \in B\left(\lambda_{k}, 3 \delta\left|\lambda_{k}\right|\right)$. Then $\left|\lambda_{k}\right|<|z|(1-3 \delta)^{-1}<2|z|, k>1$. In view of $(2.3)$ we hence get:

$$
\ln \left|\frac{z-\lambda_{k}}{3 \delta\left|\lambda_{k}\right|}\right|^{n_{k}} \geqslant-\varepsilon(2|z|)^{\rho(2|z|)} \geqslant-2^{\rho+1} \varepsilon|z|^{\rho(|z|)}, \quad z \in B\left(\lambda_{k}, 3 \delta\left|\lambda_{k}\right|\right) \backslash B, \quad k>k_{1} .
$$

Thus, we arrive at inequality (3.4).

Finally, assume that (3.4) holds. Suppose that (3.1) fails. Then for some $\varepsilon>0$ there exist sequences $\left\{w_{m}\right\},\left\{z_{m}\right\}$ such that $z_{m} \in \mathbb{C} \backslash B,\left|w_{m}\right| \rightarrow \infty$ and

$$
\ln \left|q_{\Lambda}\left(z_{m}, w_{m}, m^{-1}\right)\right|<-\varepsilon\left|z_{m}\right|^{\rho\left(\left|z_{m}\right|\right)}, \quad m \geqslant 1 .
$$

By the definition of the function $q_{\Lambda}$ this implies that for each $m \geqslant 1$, the circle $B\left(w_{m}, \frac{\left|w_{m}\right|}{m}\right)$ contains at least one of the points $\lambda_{k}$. Let $\lambda_{k(m)}$ be a point in the circle $B\left(w_{m}, \frac{\left|w_{m}\right|}{m}\right)$ located at the minimal distance from the point $z_{m}$ among all $\lambda_{k} \in B\left(w_{m}, \frac{\left|w_{m}\right|}{m}\right), m \geqslant 1$; if there are several such points, we choose arbitrarily one of them. Let us show that (3.5) contradicts the assumptions of the lemma. In order to do this, we estimate from below two groups of the factors forming the quantity $\left|q_{\Lambda}\left(z_{m}, w_{m}, m^{-1}\right)\right|$. The first one is formed by the points $\lambda_{k} \in B\left(w_{m}, \frac{\left|w_{m}\right|}{m}\right), k \neq k(m)$, while the second corresponds to the point $\lambda_{k(m)}$. Let us estimate the first group. According the assumptions, $S_{\Lambda}=0$. Then due to the definition of $S_{\Lambda}$, there exist $\delta \in\left(0, \frac{1}{3}\right)$ and $k_{0}$ such that

$$
\frac{\ln \left|q_{\Lambda}^{k}\left(\lambda_{k}, \delta\right)\right|}{\left|\lambda_{k}\right|^{\rho\left(\left|\lambda_{k}\right|\right)}} \geqslant-\frac{\varepsilon}{3}, \quad k \geqslant k_{0}
$$

We choose $m_{0}$ so that $k(m) \geqslant k_{0}, m \geqslant 2 \delta^{-1}$ and $B\left(w_{m}, \frac{\left|w_{m}\right|}{m}\right) \subset B\left(\lambda_{k(m)}, \delta\left|\lambda_{k(m)}\right|\right), m \geqslant$ $m_{0}$; the latter is possible since $\lambda_{k(m)} \in B\left(w_{m}, \frac{\left|w_{m}\right|}{m}\right)$. Let $\lambda_{k} \in B\left(w_{m}, \frac{\left|w_{m}\right|}{m}\right)$. Assume that $\left|z_{m}-\lambda_{k}\right|<2^{-1}\left|\lambda_{k(m)}-\lambda_{k}\right|$. Then according the choice of the point $\lambda_{k(m)}$ we have:

$$
\left|\lambda_{k(m)}-\lambda_{k}\right| \leqslant\left|z_{m}-\lambda_{k}\right|+\left|z_{m}-\lambda_{k(m)}\right| \leqslant 2\left|z_{m}-\lambda_{k}\right|<\left|\lambda_{k(m)}-\lambda_{k}\right| .
$$

We have obtained a contradiction. Therefore,

$$
\left|z_{m}-\lambda_{k}\right| \geqslant 2^{-1}\left|\lambda_{k(m)}-\lambda_{k}\right|, \quad \lambda_{k} \in B\left(w_{m}, \frac{\left|w_{m}\right|}{m}\right) .
$$

Taking into consideration (2.4), (3.6) and the choice of the number $m_{0}$, we obtain:

$$
\begin{aligned}
\prod_{\lambda_{k} \in B\left(w_{m}, \frac{\left|w_{m}\right|}{m}\right), k \neq k(m)}\left(\frac{\left|z_{m}-\lambda_{k}\right|}{3 m^{-1}\left|\lambda_{k}\right|}\right)^{n_{k}} \geqslant & \prod_{\lambda_{k} \in B\left(w_{m}, \frac{\left|w_{m}\right|}{m}\right), k \neq k(m)}\left(\frac{\left|\lambda_{k(m)}-\lambda_{k}\right|}{6 m^{-1}\left|\lambda_{k}\right|}\right)^{n_{k}} \\
\geqslant & \prod_{\lambda_{k} \in B\left(w_{m}, \frac{\left|w_{m}\right|}{m}\right), k \neq k(m)}\left(\frac{\left|\lambda_{k(m)}-\lambda_{k}\right|}{3 \delta\left|\lambda_{k}\right|}\right)^{n_{k}} \\
\geqslant & \prod_{\lambda_{k} \in B\left(w_{m}, \frac{\left|w_{m}\right|}{m}\right), k \neq k(m)}\left(\frac{\left|\lambda_{k(m)}-\lambda_{k}\right|}{3 \delta\left|\lambda_{k}\right|}\right)^{n_{k}} \\
= & \left|q_{\Lambda}^{k(m)}\left(\lambda_{k(m)}, \delta\right)\right| \geqslant \exp \left(-\frac{\varepsilon\left|\lambda_{k(m)}\right| \rho\left(\left|\lambda_{k(m)}\right|\right.}{3}\right)
\end{aligned}
$$


as $m \geqslant m_{0}$. Since $\lambda_{k(m)} \in B\left(w_{m}, \frac{\left|w_{m}\right|}{m}\right)$ and by $(2.5)$ and $(3.5) z_{m} \in B\left(w_{m}, \frac{5\left|w_{m}\right|}{m}\right), m \geqslant 1$, in view of (2.3) there exists a number $m_{1} \geqslant m_{0}$ such that

$$
\exp \left(-\frac{\varepsilon\left|\lambda_{k(m)}\right|^{\rho\left(\left|\lambda_{k(m)}\right|\right)}}{3}\right) \geqslant \exp \left(-\frac{\varepsilon\left|z_{m}\right|^{\rho\left(\left|z_{m}\right|\right)}}{2}\right), \quad m \geqslant m_{1} .
$$

We proceed to estimating the second group of the factors forming $\left|q_{\Lambda}\left(z_{m}, w_{m}, m^{-1}\right)\right|$ constructed by the point $\lambda_{k(m)}$. By (3.4), there exist $m_{2} \geqslant m_{1}$ and $\delta_{1} \in\left(0, \frac{1}{3}\right)$ such that

$$
\ln \left|\frac{z_{m}-\lambda_{k(m)}}{3 \delta_{1}\left|\lambda_{k(m)}\right|}\right|^{n_{k(m)}} \geqslant-\frac{\varepsilon\left|z_{m}\right| \rho(|z|)}{2}, \quad m \geqslant m_{2} .
$$

We choose $m_{3} \geqslant m_{2}$ by the condition $m^{-1} \leqslant \delta_{1}, m \geqslant m_{3}$. Then

$$
\ln \left|\frac{z_{m}-\lambda_{k(m)}}{3 m^{-1}\left|\lambda_{k(m)}\right|}\right|^{n_{k(m)}} \geqslant \ln \left|\frac{z_{m}-\lambda_{k(m)}}{3 \delta_{1}\left|\lambda_{k(m)}\right|}\right|^{n_{k(m)}} \geqslant-\frac{\varepsilon\left|z_{m}\right|^{\rho(|z|)}}{2}, \quad m \geqslant m_{3} .
$$

In view of (3.7) and (3.8) this implies

$$
\ln \left|q_{\Lambda}\left(z_{m}, w_{m}, m^{-1}\right)\right| \geqslant-\varepsilon\left|z_{m}\right|, \quad m \geqslant m_{3} .
$$

The latter contradicts (3.5). Thus, (3.1) is true and this completes the proof.

We are going to describe a wide class of sets $B$ and to provide conditions ensuring the validity of Statement 3 in Lemma 3.1. Let $\Gamma=\left\{\gamma_{k}\right\}_{k=1}^{\infty}$ be a sequence of positive numbers. We let $B(\Lambda, \Gamma)=\cup_{k=1}^{\infty} B\left(\lambda_{k}, \gamma_{k}\right)$.

Lemma 3.2. Let $\Lambda=\left\{\lambda_{k}, n_{k}\right\}$ and $\Gamma=\left\{\gamma_{k}\right\}$ satisfy a condition:

$$
\frac{n_{k}}{\left|\lambda_{k}\right| \rho\left(\left|\lambda_{k}\right|\right)} \ln \frac{\gamma_{k}}{\left|\lambda_{k}\right|} \rightarrow 0, \quad k \rightarrow \infty
$$

Then Statement 3 in Lemma 3.1 holds with $B=B(\Lambda, \Gamma)$.

Proof. Let $\delta \in\left(0, \frac{1}{3}\right), \lambda_{k} \neq 0$ and $z \in B\left(\lambda_{k}, \delta\left|\lambda_{k}\right|\right) \cap \partial B$. By (3.9) we have:

$$
\frac{1}{\left|\lambda_{k}\right|^{\rho\left(\left|\lambda_{k}\right|\right)}} \ln \left|\frac{z-\lambda_{k}}{3 \delta\left|\lambda_{k}\right|}\right|^{n_{k}} \geqslant \frac{n_{k}}{\left|\lambda_{k}\right|^{\rho\left(\left|\lambda_{k}\right|\right)}} \ln \frac{\gamma_{k}}{3 \delta\left|\lambda_{k}\right|} \geqslant \frac{n_{k}}{\left|\lambda_{k}\right| \rho\left(\left|\lambda_{k}\right|\right)} \ln \frac{\gamma_{k}}{\left|\lambda_{k}\right|} \rightarrow 0
$$

This leads us to Statement 3 in Lemma 3.1.

Remark 1. a) Suppose that $\Gamma=\left\{\gamma_{k}\right\}$ satisfies (3.9) and for $r_{k} \in(0,1), k \geqslant 1$, the relation holds:

$$
\frac{n_{k}}{\left|\lambda_{k}\right|^{\rho\left(\left|\lambda_{k}\right|\right)}} \ln r_{k} \rightarrow 0, \quad k \rightarrow \infty .
$$

Then, as one can see easily, $\left\{r_{k} \gamma_{k}\right\}$ also satisfies (3.9). In particular, if

$$
m(\Lambda)=\lim _{k \rightarrow \infty} \frac{n_{k}}{\left|\lambda_{k}\right|^{\rho\left(\left|\lambda_{k}\right|\right)}}=0,
$$

then for each $r_{k} \in\left(r_{0}, 1\right), k \geqslant 1, r_{0}>0$, condition (3.10) holds true.

b) Let $m(\Lambda)=0$ and $\gamma_{k}=n_{k}\left|\lambda_{k}\right|^{1-\rho\left(\left|\lambda_{k}\right|\right)}, k \geqslant 1$. Then $\Gamma=\left\{\gamma_{k}\right\}$ satisfies (3.9). We have

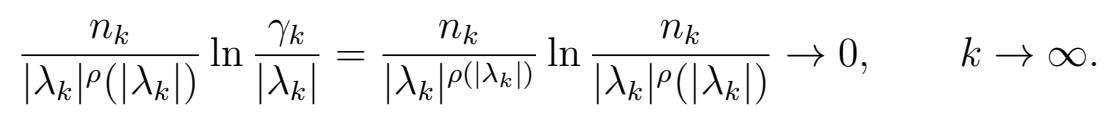

c) Assume that $n_{k} \leqslant N, k \geqslant 1$. Then (3.9) holds if

$$
\frac{\ln \gamma_{k}}{\left|\lambda_{k}\right|^{\rho\left(\left|\lambda_{k}\right|\right)}} \rightarrow 0, \quad k \rightarrow \infty .
$$


Indeed, by (2.1), for $k \geqslant k_{0}$ we have:

$$
\frac{\ln \left|\lambda_{k}\right|}{\left|\lambda_{k}\right|^{\rho\left(\left|\lambda_{k}\right|\right)}} \leqslant \frac{\ln \left|\lambda_{k}\right|}{\left|\lambda_{k}\right|^{\rho / 2}} \rightarrow 0, \quad k \rightarrow \infty
$$

This is why (3.9) is true. For instance, we can choose

$$
\gamma_{k}=\exp \left(-\varepsilon_{k}\left|\lambda_{k}\right|^{\rho\left(\left|\lambda_{k}\right|\right)}\right), \quad k \geqslant 1, \quad 0<\varepsilon_{k} \rightarrow 0, \quad k \rightarrow \infty .
$$

We let

$$
\varepsilon_{k}=\frac{1}{\left|\lambda_{k}\right|^{\rho\left(\left|\lambda_{k}\right|\right)}} \ln \frac{\left|\lambda_{k}\right|^{2 \rho\left(\left|\lambda_{k}\right|\right)}}{\tau} .
$$

If in addition $\bar{n}(\Lambda)<+\infty$, then the series

$$
\sum_{k=1}^{\infty} \gamma_{k}=\sum_{k=1}^{\infty} \frac{\tau}{\left|\lambda_{k}\right|^{2 \rho\left(\left|\lambda_{k}\right|\right)}}
$$

converges. Thus, in the case $n_{k} \leqslant N, k \geqslant 1$, and $\bar{n}(\Lambda)<+\infty$, the exceptional set $B(\Lambda, \Gamma)$ in Lemma 3.2 consists of the circles centered at the points $\lambda_{k}$ and the sum of their radii can be made arbitrarily small.

Let $\Gamma=\left\{\gamma_{k}\right\}, \Lambda=\left\{\lambda_{k}, n_{k}\right\}$. For each $k \geqslant 1$, by the symbol $\beta_{k}$ we denote the minimal distance from the point $\lambda_{k}$ to the points $\lambda_{s}, s \neq k$. We let

$$
\begin{aligned}
\Gamma_{0}(\tau)=\left\{\gamma_{k}^{0}(\tau)\right\}_{k=1}^{\infty}, \quad & \gamma_{k}^{0}(\tau)=\tau \min \left\{\gamma_{k}, \beta_{k}\right\}, \quad \tau \in\left(0,2^{-1}\right], \quad k \geqslant 1, \\
& B\left(\Lambda, \Gamma_{0}(\tau)\right)=\bigcup_{k=1}^{\infty} B\left(\lambda_{k}, \gamma_{k}^{0}(\tau)\right) .
\end{aligned}
$$

By (3.13) and the definition of the numbers $\beta_{k}$, the circles $B\left(\lambda_{k}, \gamma_{k}^{0}(\tau)\right)$ are mutually disjoint.

Lemma 3.3. Let $\Lambda=\left\{\lambda_{k}, n_{k}\right\}, m(\Lambda)=0, \tau \in\left(0,2^{-1}\right]$, and $S_{\Lambda}=0$. Assume that $\Gamma=\left\{\gamma_{k}\right\}$ satisfies (3.9) and $B=B\left(\Lambda, \Gamma_{0}(\tau)\right)$. Then Statement 3 in Lemma 3.1 holds true.

Proof. We suppose the contrary, then for some number $\varepsilon>0$ there exists sequences $\{k(m)\}$ and $\left\{z_{m}\right\}$ such that $k(m) \rightarrow \infty, z_{m} \in B\left(\lambda_{k(m)}, \frac{\left|\lambda_{k(m)}\right|}{m}\right) \cap \partial B$ and

$$
\ln \left|\frac{z_{m}-\lambda_{k(m)}}{3 m^{-1}\left|\lambda_{k(m)}\right|}\right|^{n_{k(m)}}<-\varepsilon\left|\lambda_{k(m)}\right|^{\rho\left(\left|\lambda_{k(m)}\right|\right)}, \quad m \geqslant 1 .
$$

Let $\Lambda_{1}$ be a sequence consisting of all pairs $\lambda_{k}, n_{k}$ such that $\gamma_{k}^{0}(\tau)=\tau \gamma_{k}$. We let $\Gamma_{1}=$ $\left\{\tau \gamma_{k}\right\}_{\lambda_{k}, n_{k} \in \Lambda_{1}}$. By assumption, $m(\Lambda)=0$ and hence, (3.10) holds with $r_{k}=\tau$. This is why according Remark 1.a), the sequence $\Gamma_{1}$ satisfies (3.9). Then by Lemma 3.2, the sequence $\Lambda_{1}$ satisfies Statement 3 in Lemma 2.1, where we let $B=B\left(\Lambda_{1}, \Gamma_{1}\right)$, and according this lemma, its Statement 4 holds as well. Therefore, there exists a number $m_{0}$ and a number $\delta \in\left(0, \frac{1}{3}\right)$ such that

$\ln \left|\frac{z_{m}-\lambda_{k(m)}}{3 m^{-1}\left|\lambda_{k(m)}\right|}\right|^{n_{k(m)}} \geqslant \ln \left|\frac{z_{m}-\lambda_{k(m)}}{3 \delta\left|\lambda_{k(m)}\right|}\right|^{n_{k(m)}} \geqslant-\frac{\varepsilon\left|z_{m}\right|^{\rho\left(\left|z_{m}\right|\right)}}{2}, \quad \lambda_{k(m)}, n_{k(m)} \in \Gamma_{1}, \quad m \geqslant m_{0}$.

Since $z_{m} \in B\left(\lambda_{k(m)}, \frac{\left|\lambda_{k(m)}\right|}{m}\right)$, then $\frac{\left|z_{m}\right|}{\left|\lambda_{k(m)}\right|} \rightarrow 1, m \rightarrow \infty$. This is why due to (2.3), for some number $m_{1} \geqslant m_{0}$, the inequality holds true:

$$
-\frac{\varepsilon\left|z_{m}\right|^{\rho\left(\left|z_{m}\right|\right)}}{2} \geqslant-\varepsilon\left|\lambda_{k(m)}\right|^{\rho\left(\left|\lambda_{k(m)}\right|\right)}, \quad m \geqslant m_{1}
$$

Thus,

$$
\ln \left|\frac{z_{m}-\lambda_{k(m)}}{3 m^{-1}\left|\lambda_{k(m)}\right|}\right|^{n_{k(m)}} \geqslant-\varepsilon\left|\lambda_{k(m)}\right|^{\rho\left(\left|\lambda_{k(m)}\right|\right)}, \quad \lambda_{k(m)}, n_{k(m)} \in \Gamma_{1}, \quad m \geqslant m_{1} .
$$


According the definition of numbers $\beta_{k}$, we find indices $p_{m}$ such that $\left|\lambda_{k(m)}-\lambda_{p_{m}}\right|=\beta_{k(m)}$, $m \geqslant 1$. By assumption, $S_{\Lambda}=0$. Therefore, there exist a number $m_{2} \geqslant m_{1}$ and a number $\delta_{1} \in\left(0, \frac{1}{3}\right)$, for which

$$
\frac{\ln \left|q_{\Lambda}^{p_{m}}\left(\lambda_{p_{m}}, \delta_{1}\right)\right|}{\left|\lambda_{p_{m}}\right| \rho\left(\left|\lambda_{p_{m}}\right|\right)} \geqslant-\frac{\varepsilon}{2}, \quad m \geqslant m_{2}
$$

Let $\lambda_{k(m)}, n_{k(m)} \in \Gamma_{1}$. Since $z_{m} \in B\left(\lambda_{k(m)}, \tau \beta_{k(m)}\right)$, then $\left|\lambda_{k(m)}-z_{m}\right| \geqslant \tau \beta_{k(m)}$. Hence,

$$
\frac{\left|\lambda_{k(m)}-\lambda_{p_{m}}\right|}{\left|\lambda_{k(m)}-z_{m}\right|}=\frac{\beta_{k(m)}}{\left|\lambda_{k(m)}-z_{m}\right|} \leqslant \frac{1}{\tau} \text {. }
$$

This is why, in view of the belonging $z_{m} \in B\left(\lambda_{k(m)}, \frac{\left|\lambda_{k(m)}\right|}{m}\right)$, we obtain:

$$
\left|\lambda_{k(m)}-\lambda_{p_{m}}\right| \leqslant \frac{1}{\tau}\left|\lambda_{k(m)}-z_{m}\right| \leqslant \frac{1}{\tau m}\left|\lambda_{k(m)}\right| .
$$

Due to (3.17), we can assume that $m \geqslant m_{2}$, the belonging holds $\lambda_{k(m)} \in B\left(\lambda_{p_{m}}, \delta_{1}\left|\lambda_{p_{m}}\right|\right)$ and $(m \tau)^{-1}<\delta_{1}$. Therefore, according (2.4) and the first inequality in (3.17), we get:

$$
\begin{aligned}
\ln \left|\frac{z_{m}-\lambda_{k(m)}}{3 m^{-1}\left|\lambda_{k(m)}\right|}\right|^{n_{k(m)}} & \geqslant \ln \left|\frac{\lambda_{k(m)}-\lambda_{p_{m}}}{3(\tau m)^{-1}\left|\lambda_{k(m)}\right|}\right|^{n_{k(m)}} \\
& \geqslant \ln \left|\frac{\lambda_{k(m)}-\lambda_{p_{m}}}{3 \delta_{1}\left|\lambda_{k(m)}\right|}\right|^{n_{k(m)}} \geqslant\left|q_{\Lambda}^{p_{m}}\left(\lambda_{p_{m}}, \delta_{1}\right)\right|, \quad m \geqslant m_{2} .
\end{aligned}
$$

According (3.17), $\lambda_{p_{m}} \in B\left(\lambda_{k(m)}, \frac{\left|\lambda_{k(m)}\right|}{\tau m}\right)$. Therefore, by (2.3), for some index $m_{3} \geqslant m_{2}$, the estimate holds:

$$
-\frac{\varepsilon}{2}\left|\lambda_{p_{m}}\right|^{\rho\left(\left|\lambda_{p_{m}}\right|\right)} \geqslant-\varepsilon\left|\lambda_{k(m)}\right|^{\rho\left(\left|\lambda_{k(m)}\right|\right)}, \quad m \geqslant m_{3} .
$$

In view of (3.18) and (3.16) this implies:

$$
\ln \left|\frac{z_{m}-\lambda_{k(m)}}{3 m^{-1}\left|\lambda_{k(m)}\right|}\right|^{n_{k(m)}} \geqslant-\varepsilon\left|\lambda_{k(m)}\right|^{\rho\left(\left|\lambda_{k(m)}\right|\right)}, \quad m \geqslant m_{3}
$$

Together with (3.15), this contradicts (3.14).

Remark 2. Under the assumptions of Lemma 3.3, the sequence $\Gamma=\left\{\gamma_{k}\right\}$ can be chosen so that

$$
\lim _{k \rightarrow \infty} \frac{\gamma_{k}}{\left|\lambda_{k}\right|}=0
$$

Indeed, we let $\gamma_{k}=\min \left\{\left|\lambda_{k}\right|, n_{k}\left|\lambda_{k}\right|^{1-\rho\left(\left|\lambda_{k}\right|\right)}\right\}, k \geqslant 1$. According Remark 1.b), the sequence $\Gamma=\left\{\gamma_{k}\right\}$ satisfies (3.9). Since $m(\Lambda)=0$, identity (3.19) follows.

In the end of the section, we provide a simple statement which is in some sense (in view of Lemma 3.1) inverse to Lemma 3.3.

Lemma 3.4. Let $\Lambda=\left\{\lambda_{k}, n_{k}\right\}$ and $B_{k}, k \geqslant 1$, be open mutually disjoint sets. Assume that $\lambda_{k} \in B_{k}, k \geqslant 1$,

$$
\lim _{k \rightarrow \infty} \frac{d_{k}}{\left|\lambda_{k}\right|}=0
$$

where $d_{k}$ are diameters $B_{k}$ and for each $\varepsilon>0$ there exist $k_{0}$ and $\delta \in\left(0, \frac{1}{3}\right)$ such that

$$
\ln \left|q_{\Lambda}\left(z, \lambda_{k}, \delta\right)\right| \geqslant-\varepsilon|z|^{\rho(|z|)}, \quad z \in B\left(\lambda_{k}, \delta\left|\lambda_{k}\right|\right) \cap \partial B, \quad B=\bigcup_{k=1}^{\infty} B_{k}, \quad k \geqslant k_{0} .
$$

Then $S_{\Lambda}=0$ and $m(\Lambda)=0$. 
Proof. Let $\varepsilon>0$. By (3.21), in view (2.4) and the definition of the function $q_{\Lambda}^{k}$ we have:

$$
\ln \left|q_{\Lambda}^{k}(z, \delta)\right| \geqslant-\varepsilon|z|^{\rho(|z|)}, \quad z \in B\left(\lambda_{k}, \delta\left|\lambda_{k}\right|\right) \cap \partial B, \quad k \geqslant k_{0} .
$$

According (2.3), there exist a number $k_{1} \geqslant k_{0}$ and a number $\delta_{1} \in(0, \delta)$ such that

$$
-\varepsilon|z|^{\rho(|z|)} \geqslant-2 \varepsilon\left|\lambda_{k}\right|^{\rho\left(\left|\lambda_{k}\right|\right)}, \quad z \in B\left(\lambda_{k}, \delta_{1}\left|\lambda_{k}\right|\right), \quad k \geqslant k_{1} .
$$

Therefore, in view of (2.4) we obtain:

$$
\ln \left|q_{\Lambda}^{k}\left(z, \delta_{1}\right)\right| \geqslant \ln \left|q_{\Lambda}^{k}(z, \delta)\right| \geqslant-2 \varepsilon\left|\lambda_{k}\right|^{\rho\left(\left|\lambda_{k}\right|\right)}, \quad z \in B\left(\lambda_{k}, \delta_{1}\left|\lambda_{k}\right|\right) \cap \partial B, \quad k \geqslant k_{1} .
$$

By (3.20) we can suppose that $B_{k}$ is compactly embedded into $B\left(\lambda_{k}, \delta_{1}\left|\lambda_{k}\right|\right), k \geqslant k_{1}$. The polynomial $q_{\Lambda}^{k}\left(z, \delta_{1}\right)$ has no zeroes in the set $B_{k}$. Hence, by the minimum principle for harmonic functions the latter inequality is extended on $B_{k}$. In particular,

$$
\ln \left|q_{\Lambda}^{k}\left(\lambda_{k}, \delta_{1}\right)\right| \geqslant-2 \varepsilon\left|\lambda_{k}\right|^{\rho\left(\left|\lambda_{k}\right|\right)}, \quad k \geqslant k_{1} .
$$

By (2.4),

$$
\left|q_{\Lambda}^{k}\left(\lambda_{k}, \alpha\right)\right| \geqslant\left|q_{\Lambda}^{k}\left(\lambda_{k}, \delta_{1}\right)\right|, \quad \alpha \in\left(0, \delta_{1}\right)
$$

Thus,

$$
S_{\Lambda}=\lim _{\alpha \rightarrow 0} \frac{\lim }{k \rightarrow \infty} \frac{\left.\ln \mid q_{\Lambda}^{k}\left(\lambda_{k}, \alpha\right)\right) \mid}{\left|\lambda_{k}\right|} \geqslant-2 \varepsilon .
$$

Since $\varepsilon>0$ is arbitrary, then $S_{\Lambda}=0$.

Now we are going to show that $m(\Lambda)=0$. Let $z_{k} \in \partial B_{k} \subset B\left(\lambda_{k}, \delta_{1}\left|\lambda_{k}\right|\right), k \geqslant k_{1}$. According (3.21), (3.22) and in view of (2.4) we have:

$$
\ln \left|\frac{z_{k}-\lambda_{k}}{3 \delta\left|\lambda_{k}\right|}\right|^{n_{k}} \geqslant \ln \left|q_{\Lambda}\left(z_{k}, \lambda_{k}, \delta\right)\right| \geqslant-2 \varepsilon\left|\lambda_{k}\right|^{\rho\left(\left|\lambda_{k}\right|\right)}, \quad k \geqslant k_{1} .
$$

Therefore, by (2.33) and (2.32),

$$
\frac{n_{k}}{\left|\lambda_{k}\right|^{\rho\left(\left|\lambda_{k}\right|\right)}} \ln \frac{d_{k}}{3 \delta\left|\lambda_{k}\right|} \geqslant-2 \varepsilon, \quad k \geqslant k_{1} .
$$

Now it follows from $(3.20)$ that $m(\Lambda)=0$.

\section{LOWER BOUNDS FOR ENTIRE FUNCTIONS OF COMPLETELY REGULAR GROWTH}

Let $\rho(r)$ be a proximate order and $f$ be an entire function of order at most $\rho(r)$, that is,

$$
\ln |f(z)| \leqslant A+B|z|^{\rho(|z|)}, \quad z \in \mathbb{C} .
$$

The function

$$
h_{f}(\varphi)=\varlimsup_{r \rightarrow+\infty} \frac{\ln \left|f\left(r e^{i \varphi}\right)\right|}{r^{\rho(r)}}, \quad \varphi \in[0,2 \pi],
$$

is called an indicator of $f$. It is continuous [1, Ch. I., Sect. 16]. We mention one more property of the indicator [1, Ch. I, Sect. 18, Thm. 28]: for each $\varepsilon>0$ there exists $R(\varepsilon)>0$ such that

$$
\ln \left|f\left(r e^{i \varphi}\right)\right| \leqslant\left(h_{f}(\varphi)+\varepsilon\right) r^{\rho(r)}, \quad \varphi \in[0,2 \pi], \quad r \geqslant R(\varepsilon) .
$$

A function $f$ is said to have a completely regular growth [1, Ch. III] if

$$
h_{f}(\varphi)=\lim _{r \notin E, r \rightarrow+\infty} \frac{\ln \left|f\left(r e^{i \varphi}\right)\right|}{r^{\rho(r)}}, \quad \varphi \in[0,2 \pi],
$$

where $E \subset(0,+\infty)$ is a set of relative zero measure $\left(E_{0}\right.$-set $)$, that is,

$$
\lim _{r \rightarrow+\infty} \frac{\operatorname{mes}(E \cap(0, r))}{r}=0 \text {; }
$$


here the symbol mes stands for the Lebesgue measure. A function $f$ is said to have a regular growth on the ray $L_{\varphi}=\left\{r e^{i \varphi}, r>0\right\}$ if

$$
h_{f}(\varphi)=\lim _{r \notin E_{\varphi}, r \rightarrow+\infty} \frac{\ln \left|f\left(r e^{i \varphi}\right)\right|}{r},
$$

where $E_{\varphi}$ is $E_{0}$-set. If $f$ has a regular growth on each ray, then the set $E_{\varphi}$, generally speaking, depends on $\varphi \in[0,2 \pi]$. However, it turns out that we can find an exceptional $E_{0}$-set suitable for all $\varphi \in[0,2 \pi][1$, Ch. III, Sect. 1, Thm. 1]. In other words, the function $f$ has a completely regular growth if and only if it has a regular growth on each ray. One more equivalent definition of a function of regular growth is known. It is implied by the following result.

Lemma 4.1. Let $f$ be an entire function of order at most $\rho(r)$. The following statements are equivalent:

1. The function $f$ has a regular growth on the ray $L_{\varphi}$.

2. There exists a sequence $\left\{z_{m}\right\}_{m=1}^{\infty}$ such that

$$
\begin{array}{ll}
\lim _{m \rightarrow \infty}\left|z_{m}\right|=\infty, & \lim _{m \rightarrow \infty} \frac{z_{m}}{\left|z_{m}\right|}=e^{i \varphi}, \\
\lim _{m \rightarrow \infty} \frac{\left|z_{m+1}\right|}{\left|z_{m}\right|}=1, & \lim _{m \rightarrow \infty} \frac{\ln \left|f\left(z_{m}\right)\right|}{\left|z_{m}\right| \rho\left(\left|z_{m}\right|\right)}=h_{f}(\varphi) .
\end{array}
$$

Proof. Let $f$ has a regular growth on the ray $L_{\varphi}$. Then (4.2) holds true. We form a sequence $\left\{p_{m}\right\}_{m=1}^{\infty}$ of all natural numbers $p$ such that the semi-interval $[p-1, p)$ is not entirely contained in $E_{\varphi}$. For each $m \geqslant 1$ we arbitrarily choose a number $r_{m} \in\left[p_{m}-1, p_{m}\right) \backslash E_{\varphi}$ and we obtain a sequence $\left\{z_{m}=r_{m} e^{i \varphi}\right\}_{m=1}^{\infty}$. By construction, the first and second identities in (4.3) hold, and in view of (4.2), the forth identity in (4.3) is true as well. Let us show that the third identity holds. Assume a contrary, then there exists a sequence $\left\{z_{m_{l}}\right\}_{l=1}^{\infty}$ such that for some $\alpha>1$ the inequalities hold

$$
\left|z_{m_{l}+1}\right|>\alpha\left|z_{m_{l}}\right|, \quad l \geqslant 1 .
$$

Then by construction, for each $l \geqslant 1$, the semi-interval $\left[\left(\left[\left|z_{m_{l}}\right|\right]+1\right),\left[\alpha\left|z_{m_{l}}\right|\right]\right)$ lies in the set $E_{\varphi}$, where $[x]$ denotes an integer part of a number $x$. Therefore,

$$
\varlimsup_{r \rightarrow+\infty} \frac{\operatorname{mes}\left(E_{\varphi} \cap(0, r)\right)}{r} \geqslant \varlimsup_{l \rightarrow \infty} \frac{\operatorname{mes}\left(E_{\varphi} \cap\left(0,\left[\alpha\left|z_{m_{l}}\right|\right]\right)\right)}{\left[\alpha\left|z_{m_{l}}\right|\right]} \geqslant \lim _{l \rightarrow \infty} \frac{\left[\alpha\left|z_{m_{l}}\right|\right]-\left[\left|z_{m_{l}}\right|\right]-1}{\left[\alpha\left|z_{m_{l}}\right|\right]}=\frac{\alpha-1}{\alpha} .
$$

We have obtained a contradiction with the fact that $E_{\varphi}$ is $E_{0}$-set. Thus, (4.3) is true.

Assume now that Statement 2 holds. We can assume that $\left|z_{m+1}\right| \geqslant\left|z_{m}\right|, m \geqslant 1$.

Since $h_{f}$ is a continuous function, by (2.3), for each $\varepsilon>0$ there exist numbers $\delta_{0} \in(0,1)$ and $R_{0}(\varepsilon)$ such that

$$
\begin{aligned}
& \left|t^{\rho(t)} h_{f}(\psi)-r^{\rho(r)} h_{f}(\varphi)\right| \leqslant \varepsilon r^{\rho(r)}, \quad \varphi \in[0,2 \pi], \quad t e^{i \psi} \in B\left(r e^{i \varphi}, r \delta_{0}\right), \quad r \geqslant R_{0}(\varepsilon), \\
& \left|t^{\rho(t)}-r^{\rho(r)}\right| \leqslant \varepsilon r^{\rho(r)}, \quad t e^{i \psi} \in B\left(r e^{i \varphi}, r \delta_{0}\right), \quad r \geqslant R_{0}(\varepsilon) .
\end{aligned}
$$

Let $k \geqslant 1$. We choose $\delta_{k} \in(0,1)$ such that for $\varepsilon=k^{-2}$ and $\delta_{0}=\delta_{k}$ inequalities (4.4) and (4.5) hold. According (4.3), we find an index $m(k)$, for which

$$
\begin{aligned}
& \left|\frac{z_{m}}{\left|z_{m}\right|}-e^{i \varphi}\right|<\frac{\delta_{k}}{12}, \quad \frac{\left|z_{m+1}\right|}{\left|z_{m}\right|}-1<\frac{\delta_{k}}{12}, \quad m \geqslant m(k), \\
& \frac{\ln \left|f\left(z_{m}\right)\right|}{\left|z_{m}\right| \rho\left(\left|z_{m}\right|\right)} \geqslant h_{f}(\varphi)-\frac{1}{k^{2}}, \quad m \geqslant m(k) .
\end{aligned}
$$

Let $R\left(k^{-2}\right) \geqslant R_{0}\left(k^{-2}\right)$ be defined by inequality (4.1). We can assume that

$$
\left(1-\delta_{k}\right)\left|z_{m(k)}\right| \geqslant R\left(k^{-2}\right), \quad m \geqslant m(k) .
$$


We consider the functions $f_{m}(z)=f(z)\left(f\left(z_{m}\right)\right)^{-1}, m \geqslant m(k)$. By (4.8), (4.1), (4.4), (4.5) and (4.7) we have:

$$
\begin{aligned}
\ln \left|f_{m}\left(r e^{i \psi}\right)\right| & =\ln \left|f\left(r e^{i \psi}\right)\right|-\ln \left|f\left(z_{m}\right)\right| \leqslant\left(h_{f}(\psi)+\frac{1}{k^{2}}\right) r^{\rho(r)}-\left(h_{f}(\varphi)-\frac{1}{k^{2}}\right)\left|z_{m}\right|^{\rho\left(\left|z_{m}\right|\right)} \\
& \leqslant 4 k^{-2}\left|z_{m}\right|^{\rho\left(\left|z_{m}\right|\right)}, \quad z \in B\left(z_{m}, \delta_{k}\left|z_{m}\right|\right), \quad m \geqslant m(k) .
\end{aligned}
$$

Then according theorem on lower bound for the absolute value of an analytic function in a circle [6, Ch. I, Sect. 4, Thm. 4.2] with $2 \eta=k^{-1}$, the inequality holds:

$$
\ln \left|f_{m}(z)\right| \geqslant-4 k^{-2}(2+\ln (3 e k))\left|z_{m}\right|^{\rho\left(\left|z_{m}\right|\right)}, \quad z \in B\left(z_{m}, 6^{-1} \delta_{k}\left|z_{m}\right|\right) \backslash B_{k, m},
$$

where $B_{k, m}$ is a finite set of circles with total sum of radii equalling to $(3 k)^{-1} \delta_{k}\left|z_{m}\right|$. Hence, in view of (4.4), (4.5), and (4.7), we obtain:

$$
\begin{aligned}
\ln \left|f\left(r e^{i \psi}\right)\right| \geqslant & \left(h_{f}(\varphi)-k^{-2}\right)\left|z_{m}\right|^{\rho\left(\left|z_{m}\right|\right)}-4 k^{-2}(2+\ln (3 e k))\left|z_{m}\right|^{\rho\left(\left|z_{m}\right|\right)} \\
\geqslant & \left(h_{f}(\psi) r-3 k^{-2}\right) r^{\rho(r)}-4 k^{-2}(2+\ln (6 k)) r^{\rho(r)}-k^{-2} r^{\rho(r)} \\
= & \left(h_{f}(\psi)-\varepsilon_{k}\right) r^{\rho(r)}, \\
& \varepsilon_{k}=4 k^{-2}(3+\ln (6 k)), \quad r e^{i \psi} \in B\left(z_{m}, 6^{-1} \delta_{k}\left|z_{m}\right|\right) \backslash B_{k, m} .
\end{aligned}
$$

By the second inequality in (4.6), there exists a sequence $\left\{z_{m_{k, j}}\right\}_{j=1}^{\infty}$ such that

$$
z_{m_{k, 1}}=z_{m(k)}, \quad\left(\beta_{k}\right)^{j-1}\left|z_{m(k)}\right| \leqslant\left|z_{m_{k, j}}\right|<\left(\beta_{k}\right)^{j}\left|z_{m(k)}\right|, \quad \beta_{k}=1+\frac{\delta_{k}}{12}, \quad j>1 .
$$

By the first inequality in (4.6) this implies the inclusions:

$$
\left[\left(\beta_{k}\right)^{j-1}\left|z_{m(k)}\right| e^{i \varphi}, \quad\left(\beta_{k}\right)^{j}\left|z_{m(k)}\right| e^{i \varphi}\right) \subset B\left(z_{z_{m_{k, j}}}, 6^{-1} \delta_{k}\left|z_{z_{m_{k, j}}}\right|\right), \quad j \geqslant 1 .
$$

We define sets $E_{k, j} \subset(0,+\infty)$ by the identities

$$
\left\{r e^{i \varphi}, r \in E_{k, j}\right\}=\left[\left(\beta_{k}\right)^{j-1}\left|z_{m(k)}\right| e^{i \varphi},\left(\beta_{k}\right)^{j}\left|z_{m(k)}\right| e^{i \varphi}\right) \cap B_{k, m}, \quad j \geqslant 1 .
$$

We let $E_{k}=\cup_{j=1}^{\infty} E_{k, j}$. By (4.9) we have:

$$
\ln \left|f\left(r e^{i \varphi}\right)\right| \geqslant\left(h_{f}(\varphi)-\varepsilon_{k}\right) r^{\rho(r)}, \quad r \in\left(\left|z_{m(k)}\right|,+\infty\right) \backslash E_{k} .
$$

Let $\left(\beta_{k}\right)^{j}\left|z_{m(k)}\right| \leqslant r<\left(\beta_{k}\right)^{j+1}\left|z_{m(k)}\right|$. Then in view of (4.10) we have:

$$
\begin{aligned}
\frac{\operatorname{mes}\left(E_{k} \cap(0, r)\right)}{r} & \leqslant \frac{\operatorname{mes}\left(E_{k} \cap\left(0,\left(\beta_{k}\right)^{j+1}\left|z_{m(k)}\right|\right)\right)}{\left(\beta_{k}\right)^{j}\left|z_{m(k)}\right|} \leqslant \frac{1}{\left(\beta_{k}\right)^{j}} \sum_{s=1}^{j+1} \frac{4 \delta_{k}}{3 k} \frac{\left|z_{m_{k, s}}\right|}{\left|z_{m(k)}\right|} \\
& \leqslant \frac{4 \delta_{k}}{3 k} \sum_{s=1}^{j+1} \frac{\left(\beta_{k}\right)^{s}\left|z_{m(k)}\right|}{\left(\beta_{k}\right)^{j}\left|z_{m(k)}\right|} \leqslant \frac{4 \delta_{k}\left(\beta_{k}\right)^{2}}{3 k\left(\beta_{k}-1\right)}=\frac{16\left(\beta_{k}\right)^{2}}{3 k} \leqslant \frac{16}{k} .
\end{aligned}
$$

We choose a sequence $\left\{R_{k}\right\}_{k=1}^{\infty}$ such that

$$
R_{k}>\left|z_{m(k)}\right|, \quad 16 \sum_{s=1}^{k-1} \frac{R_{s}}{s} \leqslant \frac{R_{k}}{k}, \quad k \geqslant 1 .
$$

We let

$$
\tilde{E}_{1}=E_{1} \cap\left[\left|z_{m(1)}\right|, R_{1}\right), \quad \tilde{E}_{k}=E_{k} \cap\left[R_{k-1}, R_{k}\right), \quad k>1, \quad E_{\varphi}=\bigcup_{k=1}^{\infty} \tilde{E}_{k} .
$$


Let $R_{k}<r \leqslant R_{k+1}$. Then by (4.12) and (4.13) we get

$$
\begin{aligned}
\frac{\operatorname{mes}\left(E_{\varphi} \cap(0, r)\right)}{r} & \leqslant \sum_{s=1}^{k-1} \frac{\operatorname{mes}\left(E_{s} \cap\left(0, R_{s}\right)\right)}{R_{k}}+\frac{\operatorname{mes}\left(E_{k} \cap\left(0, R_{k}\right)\right)}{R_{k}}+\frac{\operatorname{mes}\left(E_{k+1} \cap(0, r)\right)}{r} \\
& \leqslant \frac{16}{R_{k}} \sum_{s=1}^{k-1} \frac{R_{s}}{s}+\frac{16}{k}+\frac{16}{k} \leqslant \frac{33}{k} .
\end{aligned}
$$

Hence, $E_{\varphi}$ is $E_{0}$-set. Now (4.11) implies (4.2). The proof is complete.

Let $\Lambda=\left\{\lambda_{k}, n_{k}\right\}$. The sequence $\Lambda$ is said to have an angular density [1, Ch. II] (at order $\rho(r))$ if for all $\varphi_{1}<\varphi_{2}$ except possibly a countable set $\Phi_{\Lambda}$ there exists a limit

$$
n_{\Lambda}\left(\varphi_{1}, \varphi_{2}\right)=\lim _{r \rightarrow+\infty} \frac{n\left(r, \Lambda\left(\varphi_{1}, \varphi_{2}\right)\right)}{r^{\rho(r)}}
$$

where $\Lambda\left(\varphi_{1}, \varphi_{2}\right)$ the set of all pairs $\lambda_{k}, n_{k}$ such that $\lambda_{k}$ lies in the angle $\Gamma\left(\varphi_{1}, \varphi_{2}\right)=\left\{z=t e^{i \varphi}\right.$ : $\left.\varphi \in\left(\varphi_{1}, \varphi_{2}\right), t>0\right\}$, and $\Phi_{\Lambda}$ consists only of $\varphi$, for which

$$
\inf _{\alpha>0} \lim _{r \rightarrow+\infty} \frac{n(r, \Lambda(\varphi-\alpha, \varphi+\alpha))}{r^{\rho(r)}}>0 .
$$

A sequence $\Lambda$ is called a properly distributed set [1, Ch. II] at order $\rho(r)$ if it has an angular density for non-integer $\rho$, while for integer $\rho$ it additionally satisfies the Lindelöf condition: for some $b \in \mathbb{C}$, there exists the limit

$$
\Delta(\Lambda)=\lim _{r \rightarrow+\infty} r^{(\rho-\rho(r))}\left(b+\frac{1}{\rho} \sum_{\left|\lambda_{k}\right|<r} \frac{n_{k}}{\left(\lambda_{k}\right)^{\rho}}\right) .
$$

A classical result by B.Ya. Levin [1, Ch. II, Thm. 2, Ch. III, Thm. 4] states that $f$ has a regular growth if and only if its zero set $\Lambda=\left\{\lambda_{k}, n_{k}\right\}$ is properly distributed. At that, the inequality holds:

$$
\ln \left|f\left(r e^{i \varphi}\right)\right| \geqslant r^{\rho(r)} h_{f}(\varphi)+\alpha(r), \quad r e^{i \varphi} \in \mathbb{C} \backslash B_{f}, \quad \frac{\alpha(r)}{r^{\rho(r)}} \rightarrow 0, \quad r \rightarrow \infty,
$$

where $B_{f}$ is $C_{0}$-set, that is, it can be covered by circles $B\left(z_{j}, r_{j}\right), j \geqslant 1$, such that

$$
\sigma\left(B_{f}\right)=\varlimsup_{r \rightarrow \infty} \frac{1}{r} \sum_{\left|z_{j}\right| \leqslant r} r_{j}=0 .
$$

The aim of the present section is an effective constructing of an exceptional set $B_{f}$ in a form convenient for applying. In order to make such effective constructing, B.Ya. Levin introduced the notion of a regular set [1, Ch. II, Sect. 1]. This is a properly distributed set of simple points $\Lambda=\left\{\lambda_{k}, 1\right\}$ satisfying Condition (C) or (C') in Section 2 . The set $B_{\Lambda}$, which consists of the circles $B\left(\lambda_{k}, d\left|\lambda_{k}\right|^{1-\frac{\rho\left(\left|\lambda_{k}\right|\right)}{2}}\right)$ in the first case, $k \geqslant 1$, of the circles $B\left(\lambda_{k}, d^{\prime}\left|\lambda_{k(p)}\right|^{1-\rho\left(\left|\lambda_{k(p)}\right|\right)}\right)$, $d^{\prime} \leqslant \frac{d}{2}, k \geqslant 1$, in the second case, is called a $R$-set. We note that a $R$-set is not a $C_{0}$-set. Its upper linear density $\sigma\left(B_{\Lambda}\right)$ is non-zero. However, under Condition (C'), by choosing $d>0$, the quantity $\sigma\left(B_{\Lambda}\right)$ can be made arbitrarily small. Under Condition (C), the sum of areas of exceptional circles can be made arbitrarily small. B.Ya. Levin proved [1, Ch. II, Sect. 1, Thm. $5]$ that in the case as the zero set of the function $f$ is a $R$-set, as an exceptional set $B_{f}$, the set $B_{\Lambda}$ can serve in inequality (4.14).

A simple exceptional set in estimate (4.14) consisting of mutually disjoint circles arises owing to Conditions (C) and (C'), which separate simple points $\lambda_{k}$. In the present work we employ an essentially weaker condition allowing us to separate appropriately the points $\lambda_{k}$ even in the case of multiple points. This condition is just vanishing of the condensation index $S_{\Lambda}$. 
A properly distributed set $\Lambda$ is called properly balanced if the condition $S_{\Lambda}=0$ holds.

Let us establish a connection between the notions of regular and properly balanced set. We mention first that in distinction to a properly balanced set, the notion of a regular set is defined only for simple points $\lambda_{k}$.

We consider an example from work [3]. We denote $\Lambda_{1}=\left\{\lambda_{k}, 1\right\}_{k=1}^{\infty}$ and we let $\lambda_{2 k}=k$ and $\lambda_{2 k-1}=k-e^{-\varepsilon(k) k}, k \geqslant 1$, where $0<\varepsilon(k) \rightarrow 0, e^{-\varepsilon(k) k} \rightarrow 0, k \rightarrow \infty$ (for instance, $\left.\varepsilon(k)=\frac{1}{\sqrt{k}}\right)$. We also let $\Lambda_{2}=\left\{-\lambda_{k}, 1\right\}_{k=1}^{\infty}$. It is easy to see that the sequence $\Lambda=\Lambda_{1} \cup \Lambda_{2}$ has an angular density at order $\rho(r) \equiv 1$ and satisfies the Lindelöf condition, that is, this is a properly distributed set at order $\rho(r) \equiv 1$. It was proved in work [3] that the identity $S_{\Lambda_{1}}=0$ holds true. This implies easily that $S_{\Lambda}=0$, i.e., $\Lambda$ is a properly balanced set. At that, $\Lambda$ is not a regular set since $\lambda_{2 k}-\lambda_{2 k-1}=e^{-\varepsilon(k) k} \rightarrow 0, k \rightarrow \infty$ and Conditions (C) and (C') fail.

Thus, a properly balanced set (in the case of simple points) is not necessarily a regular set. The following inverse statements holds; it is implied by the examples considered in the second section.

Theorem 4.2. Let $\Lambda=\left\{\lambda_{k}, 1\right\}$ be a regular set. Then $\Lambda$ is a properly balanced set.

Theorem 4.2 means that the notion of a properly balanced set is more general than the notion of a regular set. In what follows we shall show that both notions are introduced due to the same way.

Theorem 4.3. Let $f$ be an entire function of order at most $\rho(r)$ and of a completely regular growth, $\Lambda=\left\{\lambda_{k}, n_{k}\right\}$ be its multiple zero set. Assume that $\Gamma=\left\{\gamma_{k}\right\}$ satisfies (3.9), $\tau \in\left(0, \frac{1}{2}\right]$ and $S_{\Lambda}=0$. Then (4.14) holds, where $B_{f}=B\left(\Lambda, \Gamma_{0}(\tau)\right)$.

Proof. By the assumption, the function $f$ has a completely regular growth. Therefore, according the aforementioned result by B.Ya. Levin, $\Lambda$ is a properly distributed set. In particular, $\Lambda$ has an angular density. This implies that $\Lambda$ has a density, that is, there exists a limit

$$
\lim _{r \rightarrow \infty} \frac{n(r, \Lambda)}{r^{\rho(r)}}=n(\Lambda)<+\infty .
$$

Hence,

$$
\lim _{k \rightarrow \infty} \frac{n_{k}}{\left|\lambda_{k}\right|^{\rho\left(\left|\lambda_{k}\right|\right)}} \leqslant \lim _{k \rightarrow \infty} \frac{n\left(\left|\lambda_{k}\right|+1, \Lambda\right)-n\left(\left|\lambda_{k}\right|, \Lambda\right)}{\left|\lambda_{k}\right|^{\rho\left(\left|\lambda_{k}\right|\right)}}=\lim _{k \rightarrow \infty} \frac{n\left(\left|\lambda_{k}\right|+1, \Lambda\right)}{\left(\left|\lambda_{k}\right|+1\right)^{\rho\left(\left|\lambda_{k}\right|\right)}}-\lim _{k \rightarrow \infty} \frac{n\left(\left|\lambda_{k}\right|, \Lambda\right)}{\left|\lambda_{k}\right|^{\rho\left(\left|\lambda_{k}\right|\right)}}=0 .
$$

We have obtained the identity $m(\Lambda)=0$. Thus, all assumptions of Lemma 3.3 are satisfied. According this lemma and Lemma 3.1, for each $\varepsilon>0$ there exist numbers $R_{0}>0$ and $\delta \in\left(0, \frac{1}{3}\right)$ such that

$$
\ln \left|q_{\Lambda}(z, w, \delta)\right| \geqslant-\frac{\varepsilon|z|^{\rho(|z|)}}{4}, \quad z \in B(w, \delta|w|) \backslash B\left(\Lambda, \Gamma_{0}(\tau)\right), \quad|w| \geqslant R_{0}
$$

Now we find $\delta_{0} \in(0, \delta)$ and $R_{0}(\varepsilon) \geqslant R_{0}$ such that inequalities (4.4) and (4.5) hold true.

By the assumption, the function $f$ has a completely regular growth. In particular, (4.14) holds, where $B_{f}$ is covered by circles $B\left(z_{j}, r_{j}\right)$ obeying (4.15). Hence, there exists $R_{1} \geqslant R_{0}(\varepsilon)$ such that

$$
\frac{1}{r} \sum_{\left|z_{j}\right| \leqslant 2 r} r_{j} \leqslant \frac{\delta_{0}}{3}, \quad r \geqslant R_{1} .
$$

According (4.14), we can assume that

$$
\alpha(r) \geqslant-\frac{\varepsilon r^{\rho(r)}}{4}, \quad r \geqslant\left(1-\delta_{0}\right) R_{1} .
$$


Let $|z| \geqslant R_{1}$ and a circle $B\left(z_{j}, r_{j}\right)$ intersects the circle $4 B\left(z, \delta_{0}|z|\right)$ at a point $w$. Assume that $\left|z_{j}\right|>|z|$. Then by (4.17)

Therefore,

$$
\frac{r_{j}}{\left|z_{j}\right|} \leqslant \frac{1}{\left|z_{j}\right|} \sum_{\left|z_{s}\right| \leqslant\left|z_{j}\right|} r_{s} \leqslant \frac{\delta_{0}}{3} .
$$

$$
\left(1-\frac{\delta_{0}}{3}\right)\left|z_{j}\right|<|w|<\left(1+\delta_{0}\right)|z|
$$

In view of the inequality $\delta_{0}<\frac{1}{3}$ this implies that $\left|z_{j}\right|<2|z|$. Thus, according (4.17), the sum $d$ of the diameters of all circles $B\left(z_{j}, r_{j}\right)$ intersecting $B\left(z, \delta_{0}|z|\right)$ satisfies the estimate

$$
\frac{d}{|z|} \leqslant \frac{1}{|z|} \sum_{\left|z_{j}\right| \leqslant 2|z|} 2 r_{j} \leqslant \frac{2 \delta_{0}}{3} .
$$

This is why there exists $t \in(0,1)$ such that the circumference $S\left(z, t \delta_{0}|z|\right)$ does not intersect the set $B_{f}$. We let $z=r_{0} e^{i \varphi_{0}}$. Then by (4.14), (4.4), (4.5) and (4.18) we have:

$$
\begin{aligned}
\ln \left|f\left(r e^{i \varphi}\right)\right| & \geqslant r^{\rho(r)} h_{f}(\varphi)+\alpha(r) \geqslant r_{0}^{\rho\left(r_{0}\right)} h_{f}\left(\varphi_{0}\right)-\frac{\varepsilon r_{0}^{\rho\left(r_{0}\right)}}{4}-\frac{\varepsilon r^{\rho(r)}}{4} \\
& \geqslant r_{0}^{\rho\left(r_{0}\right)} h_{f}\left(\varphi_{0}\right)-\frac{3 \varepsilon}{4} r_{0}^{\rho\left(r_{0}\right)}, \quad r e^{i \varphi} \in S\left(z, t \delta_{0}|z|\right) .
\end{aligned}
$$

We consider the function $g(\lambda)=f(\lambda)\left(q_{\Lambda}\left(\lambda, z, \delta_{0}\right)\right)^{-1}$. It is analytic in the circle $B\left(z, \delta_{0}|z|\right)$ and by (2.4), (4.19) it satisfies the estimate

$$
\ln \left|g\left(r e^{i \varphi}\right)\right| \geqslant r_{0}^{\rho\left(r_{0}\right)} h_{f}\left(\varphi_{0}\right)-\frac{3 \varepsilon}{4} r_{0}^{\rho\left(r_{0}\right)}, \quad r e^{i \varphi} \in S\left(z, t \delta_{0}|z|\right)
$$

Since $g$ has no zeroes in the ball $B\left(z, \delta_{0}|z|\right)$, by the minimum principle for harmonic functions we get:

$$
\ln |g(z)| \geqslant r_{0}^{\rho\left(r_{0}\right)} h_{f}\left(\varphi_{0}\right)-\frac{3 \varepsilon}{4} r_{0}^{\rho\left(r_{0}\right)} .
$$

Let $z \in B\left(\Lambda, \Gamma_{0}(\tau)\right)$ and $|z| \geqslant R_{2}$. Then by (4.16) and (4.20) and in view of (2.4) we have:

$$
\begin{aligned}
\ln |f(z)| & =\ln |g(z)|+\ln \left|q_{\Lambda}\left(z, z, \delta_{0}\right)\right| \geqslant \ln |g(z)|+\ln \left|q_{\Lambda}(z, z, \delta)\right| \\
& \geqslant|z|^{\rho(|z|)} h_{f}\left(\varphi_{0}\right)-\frac{3 \varepsilon}{4}|z|^{\rho(|z|)}-\frac{\varepsilon|z|^{\rho(|z|)}}{4}=|z|^{\rho(|z|)} h_{f}\left(\varphi_{0}\right)-\varepsilon|z|^{\rho(|z|)} .
\end{aligned}
$$

Since $\varepsilon>0$ is arbitrary, this gives a required statement.

Remark 3. a) As it has been mentioned, under the assumptions of Theorem 4.3, the identity $m(\Lambda)=0$ holds. Therefore, according Remark 2, the sequence $\Gamma=\left\{\gamma_{k}\right\}$ in Theorem 4.3 can be chosen so that (3.19) holds.

b) The set $B\left(\Lambda, \Gamma_{0}(\tau)\right)$ is the union of mutually disjoint circles $B\left(\lambda_{k}, \gamma_{k}^{0}(\tau)\right)$, where

$$
\gamma_{k}^{0}(\tau)=\tau \min \left\{\gamma_{k}, \beta_{k} / 2\right\}, \quad \beta_{k}=\min _{s \neq k}\left|\lambda_{k}-\lambda_{s}\right|, \quad k \geqslant 1, \lim _{k \rightarrow \infty} \frac{n_{k}}{\left|\lambda_{k}\right| \rho\left(\left|\lambda_{k}\right|\right)} \ln \frac{\gamma_{k}}{\left|\lambda_{k}\right|}=0 .
$$

According Remark 1.c), for simple points with $n_{k}=1$, the latter identity is equivalent to the relation

$$
\frac{\ln \gamma_{k}}{\left|\lambda_{k}\right|^{\rho\left(\left|\lambda_{k}\right|\right)}} \rightarrow 0, \quad k \rightarrow \infty
$$

In this case, by this remark, the sum of radii of exceptional circles $B\left(\lambda_{k}, \gamma_{k}^{0}(\tau)\right)$ can be made arbitrarily small. In particular, in view of Theorem 4.2, this can be made as $\Lambda$ is a regular set. We mention that an exceptional set in Theorem 5 in [1, Ch. II, Sect. 1] is essentially larger and it possesses only a finite upper linear density $\sigma\left(B_{f}\right)$. 
Theorem 4.4. Let $f$ be an entire function of order at most $\rho(r), \Lambda=\left\{\lambda_{k}, n_{k}\right\}$ be its multiple zero set and $B_{k}, k \geqslant 1$, be open mutually disjoint sets, $B=\cup_{k=1}^{\infty} B_{k}$. Assume that $\lambda_{k} \in B_{k}$, $k \geqslant 1$, the diameters $d_{k}$ of the sets $B_{k}$ satisfies (3.20) and that for each $\varepsilon>0$ there exists $r(\varepsilon)>0$ such that

$$
\ln \left|f\left(r e^{i \varphi}\right)\right| \geqslant\left(h_{f}(\varphi)-\varepsilon\right) r^{\rho(r)}, \quad r e^{i \varphi} \in \partial B \backslash B(0, r(\varepsilon)) .
$$

Then $S_{\Lambda}=0$ and $m(\Lambda)=0$.

Proof. Let us prove inequality (3.21). We fix $\varepsilon>0$ and choose numbers $\delta_{0} \in(0,1)$ and $R_{0}(\varepsilon) \geqslant r(\varepsilon)$ such that (4.4) and (4.5) hold. We choose $R(\varepsilon) \geqslant R_{0}(\varepsilon)$ such that (4.1) is true. Finally, we choose a number $k_{0}$ such that

$$
\left|\lambda_{k}\right|>R(\varepsilon)\left(1-\delta_{0}\right)^{-1}, \quad k \geqslant k_{0} .
$$

Let $k \geqslant k_{0}$ and $\lambda_{k}=r_{k} e^{i \varphi_{k}}$. We consider a function $g(z)=f(z)\left(q_{\Lambda}\left(z, \lambda_{k}, \frac{\delta_{0}}{5}\right)\right)^{-1}$. By $(2.5)$, (4.1), (4.22), (4.4) and (4.5) we have

$$
\ln \left|g\left(r e^{i \varphi}\right)\right| \leqslant \ln \left|f\left(r e^{i \varphi}\right)\right| \leqslant\left(h_{f}(\varphi)+\varepsilon\right) r^{\rho(r)} \leqslant\left(h_{f}\left(\varphi_{k}\right)+2 \varepsilon\right) r_{k}^{\rho\left(r_{k}\right)}, \quad r e^{i \varphi} \in S\left(\lambda_{k}, \delta_{0}\left|\lambda_{k}\right|\right) .
$$

By the minimum principle for subharmonic functions this yields:

$$
\ln \left|g\left(r e^{i \varphi}\right)\right| \leqslant\left(h_{f}\left(\varphi_{k}\right)+2 \varepsilon\right) r_{k}^{\rho\left(r_{k}\right)}, \quad r e^{i \varphi} \in B\left(\lambda_{k}, \delta_{0}\left|\lambda_{k}\right|\right) .
$$

Let $r e^{i \varphi} \in B\left(\lambda_{k}, \frac{\delta_{0}\left|\lambda_{k}\right|}{5}\right)$. Then $r>R(\varepsilon)$ and $\lambda_{k} \in B\left(r e^{i \varphi}, \delta_{0} r\right)$. Therefore, in view of (4.4) and (4.5), we have

$$
\ln \left|g\left(r e^{i \varphi}\right)\right| \leqslant\left(h_{f}(\varphi)+4 \varepsilon\right) r^{\rho(r)} .
$$

By (4.21) this implies that for all $k \geqslant k_{0}$ the inequality holds:

$$
\ln \left|q_{\Lambda}\left(r e^{i \varphi}, \lambda_{k}, \frac{\delta_{0}}{5}\right)\right|=\ln \left|f\left(r e^{i \varphi}\right)\right|-\ln \left|g\left(r e^{i \varphi}\right)\right| \geqslant-5 \varepsilon r^{\rho(r)}, \quad r e^{i \varphi} \in B\left(\lambda_{k}, \frac{\delta_{0}\left|\lambda_{k}\right|}{5}\right) \cap \partial B .
$$

This gives (3.21). Thus, in view of the assumptions of the theorem, all assumptions of Lemma 3.4 are satisfied. Hence, its statement is true and $S_{\Lambda}=m(\Lambda)=0$.

Theorem 4.5. Let $f$ be an entire function of order at most $\rho(r)$ and $\Lambda=\left\{\lambda_{k}, n_{k}\right\}$ is its multiple zero set. The following statements are equivalent:

1. $\Lambda$ is a properly balanced set.

2. There exist numbers $\gamma_{k}^{0}>0, k \geqslant 1$, such that $\frac{\gamma_{k}^{0}}{\left|\lambda_{k}\right|} \rightarrow 0, k \rightarrow \infty$, the circles $B\left(\lambda_{k}, \gamma_{k}^{0}\right)$ are mutually disjoint and (4.14) holds, where $B_{f}=\cup_{k=1}^{\infty} B\left(\lambda_{k}, \gamma_{k}^{0}\right)$.

Proof. Assume that Statement 1 is true. Then $S_{\Lambda}=0$ and $\Lambda$ is a properly distributed set. According the above result by B.Ya. Levin, the function $f$ has a completely regular growth. Thus, by Theorem 4.3 and Remark 3.a), Statement 2 holds.

Assume that Statement 2 holds. Then by Theorem 4.4 we have $S_{\Lambda}=0$. Let us show that $f$ is a function of a completely regular growth. As it has been observed in the beginning of the section, it is sufficient to show that $f$ has a regular growth on each ray $L_{\varphi}$.

We form a sequence $\left\{p_{m}\right\}_{m=1}^{\infty}$ of all natural numbers $p$ such that the semi-interval $[(p-$ 1) $\left.e^{i \varphi}, p e^{i \varphi}\right)$ does not lie entirely in $B_{f}$. For each $m \geqslant 1$, we arbitrarily choose a point $z_{m} \in$ $\left[\left(p_{m}-1\right) e^{i \varphi}, p_{m} e^{i \varphi}\right) \backslash B_{f}$ and we obtain a sequence $\left\{z_{m}\right\}_{m=1}^{\infty}$. By construction, the first and second identity in (4.3) and in view of (4.14), the fourth identity in (4.3) holds as well. Let us show that also the third identity is true. Assume a contrary, then there exists a subsequence $\left\{z_{m_{j}}\right\}_{j=1}^{\infty}$ such that for some $\alpha>1$ the inequalities

$$
\left|z_{m_{j}+1}\right|>\alpha\left|z_{m_{j}}\right|, \quad j \geqslant 1,
$$

hold. Then by construction, for each $j \geqslant 1$, the semi-interval $\left[\left(\left[\left|z_{m_{j}}\right|\right]+1\right) e^{i \varphi},\left[\alpha\left|z_{m_{j}}\right|\right] e^{i \varphi}\right)$ is contained in $B_{f}$; we recall that $[x]$ is an integer part of a number $x$. Since $B\left(\lambda_{k}, \gamma_{k}^{0}\right)$ are mutually 
disjoint, this semi-interval is contained in some circle $B\left(\lambda_{k_{j}}, \gamma_{k_{j}}^{0}\right)$. Since $\frac{\gamma_{k}^{0}}{\left|\lambda_{k}\right|} \rightarrow 0$, then $\frac{\left|w_{j}\right|}{\left|\lambda_{k_{j}}\right|} \rightarrow 1$, $j \rightarrow \infty$, where $w_{j}=\left(\left[\left|z_{m_{j}}\right|\right]+1\right) e^{i \varphi}$. This is why

$$
0=\lim _{j \rightarrow \infty} \frac{2 \gamma_{k_{j}}^{0}}{\left|\lambda_{k_{j}}\right|} \geqslant \lim _{j \rightarrow \infty} \frac{\left[\alpha\left|z_{m_{j}}\right|\right]-\left[\left|z_{m_{j}}\right|\right]-1}{\left|\lambda_{k_{j}}\right|}=\lim _{j \rightarrow \infty} \frac{\left.\left[\alpha\left|z_{m_{j}}\right|\right]-\left[\mid z_{m_{j}}\right] \mid\right]-1}{\left|w_{j}\right|}=\alpha-1>0 .
$$

We have obtained a contradiction. Thus, identities (4.3) hold and by Lemma 4.1, the function $f$ has a completely regular growth on the ray $L_{\varphi}$.

Remark 4. By Theorem 4.2, a regular set is a particular case of a properly balanced set. The first appeared with the only aim to make the exceptional set in (4.14) consisting of mutually intersecting circles centered at zero with relatively small radii. According Theorem 4.5, a necessary and sufficient condition of this is a proper balancing of the zero set of the function $f$. Thus, the notion of a properly balanced set is a natural generalization of the notion of a regular set. We also note that then notion of a properly balanced set is effective for a multiple zero set.

\section{BIBLIOGRAPHY}

1. B.Ya. Levin. Distribution of zeros of entire functions. Gostekhizdat, Moscow (1956). [Amer. Math. Soc., Providence, RI (1980).]

2. A.S. Krivosheev. A fundamental principle for invariant subspaces in convex domains // Izv. RAN. Ser. Matem. 68:2, 71-136 (2004). [Izv. Math. 68:2, 291-353 (2004).]

3. O.A. Krivosheyeva. Singular points of the sum of a series of exponential monomials on the boundary of the convergence domain // Alg. Anal. 23:2, 162-205 (2011). [St.-Petersburg Math. J. 23:2, 321350 (2012).]

4. A.S. Krivosheev, O.A. Krivosheeva. A basis in invariant subspace of entire functions // Alg. Anal. 27:2, 132-195 (2015). [St. Petersburg Math. J. 27:2, 273-316 (2016).]

5. O.A. Krivosheeva, A.S. Krivosheev. Singular points of the sum of a Dirichlet series on the convergence line // Funkts. Anal. Pril. 49:2, 54-69 (2015) [Funct. Anal. Appl. 49:2, 122-134 (2015).]

6. A.F. Leont'ev. Entire functions. Exponential series. Nauka, Moscow (1983). (in Russian).

Olesya Aleksandrovna Krivosheeva,

Bashkir State University,

Zaki Validi str. 32,

450076, Ufa, Russia

E-mail: kriolesya20060yandex.ru

Alexander Sergeevich Krivosheev,

Institute of Mathematics,

Ufa Federal Research Center, RAS,

Chernyshevsky str. 112,

450008, Ufa, Russia

E-mail: kriolesya2006@yandex.ru

Azar Il'gizovich Rafikov,

Bashkir State University,

Zaki Validi str. 32,

450076, Ufa, Russia

E-mail: azat@rafikov.me 